\title{
RECONSTRUCTION OF DENSITIES FROM COMPTON PROFILES WITH APPLYING JACOBI POLYNOMIALS
}

\author{
G. KONTRYM-SzNAJD ${ }^{a}$, M. SAMSEL ${ }^{a}$ AND R.N. WEST ${ }^{b}$ \\ ${ }^{a}$ W. Trzebiatowski Institute for Low Temperature and Structure Research \\ Polish Academy of Sciences, P.O. Box 937, 50-950 Wrocław 2, Poland \\ ${ }^{b}$ Physics Department, University of Texas-Arlington, Arlington, TX 76019, USA
}

The advent of synchrotron sources has led to an increasing availability of high resolution Compton profiles $J\left(p_{z}\right)$ and a consequent renewed interest in the reconstruction of the corresponding full momentum densities $\rho(p)$. We present results of applying a new method in which the radial parts of $\rho(p)$ and the measured profiles are expressed in terms of the Jacobi polynomials. The technique is demonstrated using model projections that correspond to $\mathrm{Mg}$ and $\mathrm{Gd}$ spectra. Reconstructed densities, being in very good agreement with model ones, are a very good performance of our new reconstruction algorithm.

PACS numbers: $71.20 .-\mathrm{b}, 78.70 . \mathrm{Bj}$

\section{Introduction}

Electronic densities in the momentum space, $\rho(p)$, can be experimentally determined by measuring Compton profiles (CP) or angular correlation of positron annihilation radiation (ACPAR) spectra. In the case of CP or one-dimensional ACPAR measurements one obtains plane projections of $\rho(\boldsymbol{p})$ :

$$
J\left(p_{z}\right)=\int_{-\infty}^{\infty} \mathrm{d} p_{x} \mathrm{~d} p_{y} \rho(\boldsymbol{p}) .
$$

The knowledge of $J\left(p_{z}\right)$ for various orientations of $p_{z}$ allows to reproduce $\rho(p)$ from measured profiles. Up to now four various algorithms have been known: Mijnarends [1] and Fourier transform [2] methods, orthogonal polynomial expansion [3] and application of maximum entropy [4]. So far, only two first algorithms $[1,2]$ have been applied to the experimental data. In this paper we present the results of usage of a new technique that is proposed in the paper [5] and shortly described below.

Both quantities $\rho(p)$ and $J\left(p_{z}\right)$ are expanded into lattice harmonics $F_{l \nu}(\Theta, \varphi)$ :

$$
\rho(p)=\sum_{l \nu} \rho_{l \nu}(p) F_{l \nu}(\Theta, \varphi)
$$




$$
J\left(p_{z}\right) \equiv J_{\beta \alpha}(p)=\sum_{l \nu} g_{l \nu}(p) F_{l \nu}(\beta, \alpha),
$$

where $F_{l \nu}$ form an orthogonal set of linear combinations of spherical harmonics $Y_{l}^{m}$ of the order $l$. Index $\nu$ distinguishes the harmonics of the same order and $(\beta, \alpha)$ describe the polar and azimuthal angles of the $p_{z}$-axis with respect to the reciprocal lattice system. In order to make our formulae clearer, the index $\nu$ will be omitted, keeping in mind that for some $l$ a few lattice harmonics of the same order have to be included. If radial parts of measured spectra are given as series of Jacobi polynomials

$$
g_{l}(p)=\sum_{k=0}^{\infty} a_{l k}\left(1-p^{2}\right) P_{l+2 k}^{(1,1)}(p)
$$

then the radial parts of a density are described by the following expression:

$$
\rho_{l}(p)=\frac{1}{\pi} \sum_{k=0}^{\infty} a_{l k}(1+2 k+l) p^{l} P_{k}^{(0, l+1 / 2)}\left(2 p^{2}-1\right) .
$$

This new algorithm has some advantages in comparison with other reconstruction techniques. First of all, the expansion of measured spectra into orthogonal polynomials has least-squares approximation properties and hence properly takes into account experimental errors - for more details see [5].

We have performed tests of this method for two different models of $\rho(p)$ that simulate electron-positron densities in $\mathrm{Mg}$ and $\mathrm{Gd}$.

\section{Reconstruction from plane projections}

Our model projections have been calculated for two models of $\rho(p)$ with hexagonal symmetry. Having 2D ACPAR data (line projections) for $\mathrm{Mg}[6]$ and Gd [7], we reconstructed $\rho(p)$ applying the Cormack method [8]. This way we had densities which were real, contained experimental errors and were smeared by the finite resolution function of the equipment (about 0.1 a.u. (atomic units), i.e. comparable with Compton profiles obtained using synchrotron sources). For these densities, treated as model ones, plane projections $J\left(p_{z}\right)$ were calculated for various orientations of $p_{z}$.

The first model of $\rho(\boldsymbol{p})$, resembling electron-positron densities in $\mathrm{Mg}$, is described by $\rho(p)=\rho_{0}(p, \Theta)+\rho_{6}(p, \Theta) \cos (6 \varphi)$. It is equivalent to description of $\rho(p)$ by two density components $\left(\rho_{0}(p)\right.$ and $\left.\rho_{6}(p)\right)$ which have various values on succeeding planes perpendicular to the [0001] direction. As in case of simple metals; $\rho(p)$ has high values for $p<p_{\mathrm{F}}$, where $p_{\mathrm{F}}$ denotes the Fermi momentum in the extended zone scheme, and very small outside the Fermi surface (FS). Our model FS is almost isotropic having very small distortions from sphericity being the highest around the $A L H$ plane (reduction of holes and electrons around the symmetry point $H$ and $L$, respectively). There is also a small contribution of the Umklapp components (in the $\Gamma M$ direction) which has the highest values on the first basal $\Gamma M K$ plane.

For this model $\rho(p)$ was reconstructed from two sets (7 and 9) plane projections with $p_{z}$ along: (1) 3 main $(\Gamma M, \Gamma K, \Gamma A)$ and four intermediate directions 
and (2) 9 intermediate ones. Application of 9 projections $\left(\beta=7.5^{\circ}, 22.5^{\circ}, 37.5^{\circ}\right.$ and $\alpha=15^{\circ}, \beta=52.5^{\circ}, 67.5^{\circ}, 82.5^{\circ}$ and $\alpha=7.5^{\circ}$ and $\left.22.5^{\circ}\right)$ with the change of $\alpha$ and $\beta$ equal to $15^{\circ}$ (instead of $30^{\circ}$ as for 7 projections) gives much better results. First of all some anisotropy of the FS on the $\Gamma M K$ plane, obtained for 7 projections, disappeared. $\rho(p)$ reconstructed from 9 projections, compared with model densities on the $\Gamma M K$ and $A L H$ planes, is displayed in Fig. 1a. The anisotropic part of $\rho(p)$, equal to $\rho_{6}(p, \Theta) \cos (6 \varphi)$ (model) and $\sum \rho_{l}(p) P_{l}^{6}(\theta) c_{l}^{6} \cos (6 \varphi)$ with
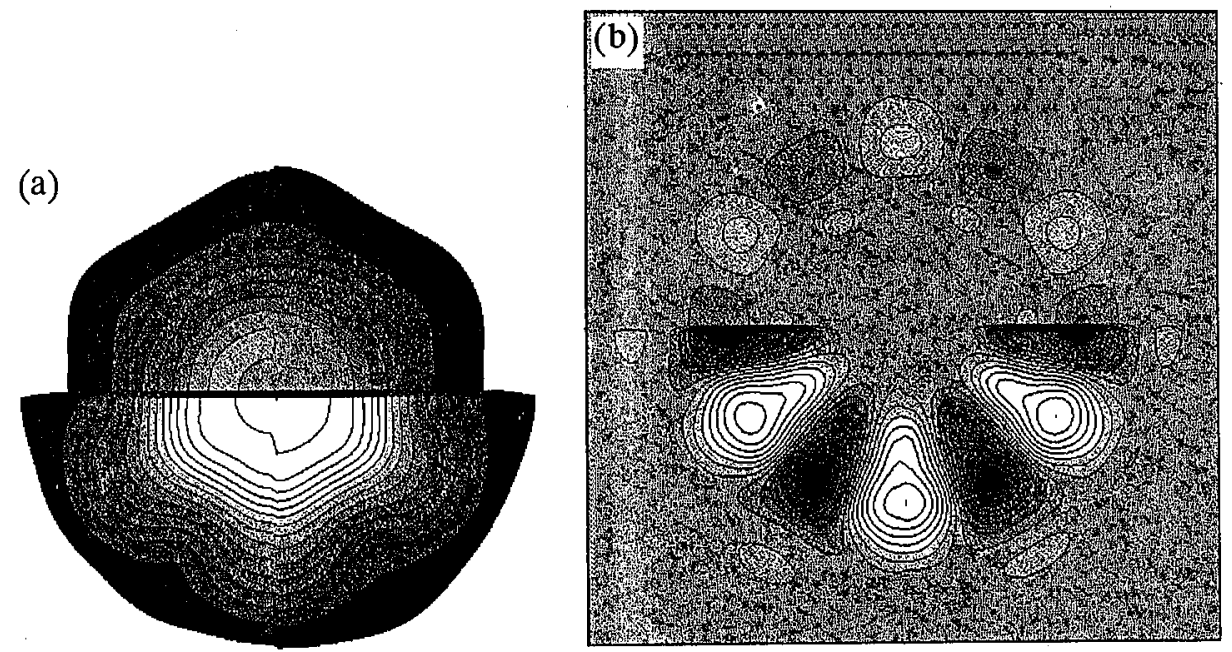

Fig. 1. Densities for $\mathrm{Mg}$ reconstructed from 9 plane projections (right part of the figure) compared with model densities (left part). Densities on the $\Gamma M K$ and $A L H$ planes are presented on the lower and upper part of the figure, respectively. Parts (a) and (b) show total densities $\varrho(p)$ and their anisotropic components, respectively.

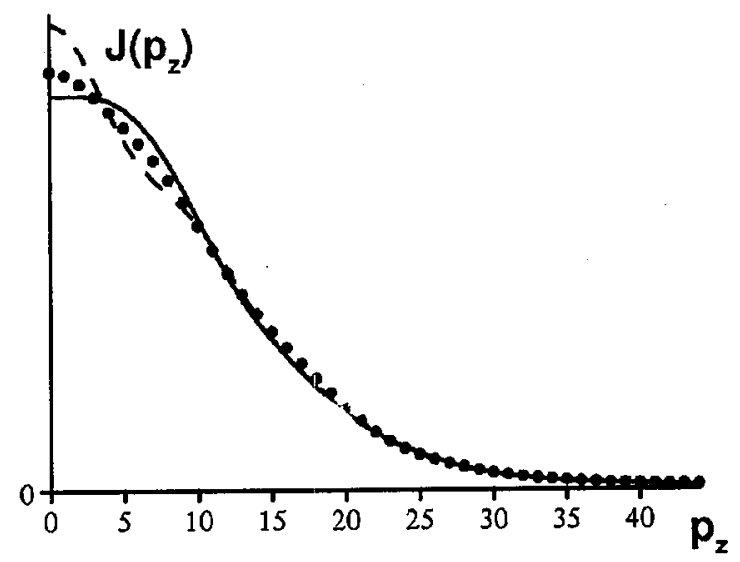

Fig. 2. Plane projections $J\left(p_{z}\right)$ for three various orientations of $p_{z}$ described by $(\beta, \alpha)$ : $\left(0^{\circ}, 0^{\circ}\right)$ - dashed line; $\left(30^{\circ}, 30^{\circ}\right)$ - dots and $\left(60^{\circ}, 15^{\circ}\right)$ - solid line. 

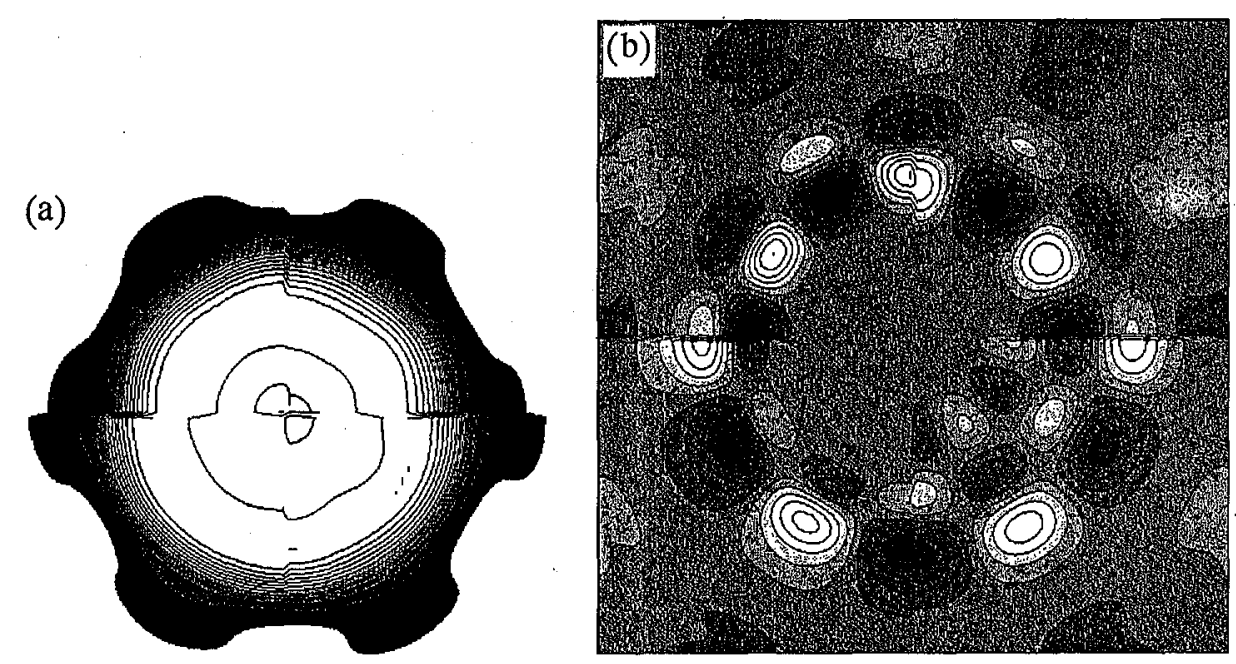

Fig. 3. The same as in Fig. 1 but for Gd after using 15 plane projections and on the planes $\Gamma M K$ (lower part) and $\Gamma^{1} M^{1} K^{-1}$ (upper part).

$l=6,8,10$ (reconstructed densities) is presented in Fig. 1 b. $P_{l}^{6}(\theta) c_{l}^{6} \cos (6 \varphi)$ denotes those lattice harmonics which distinguish various directions on the chosen hexagonal plane. Some small differences between the model and reconstructed densities, observed for small momenta, are connected with the fact that inaccuracies in the reproduction of $\rho(\boldsymbol{p})$ for higher momenta are accumulated for smaller momenta.

The second model, described by $\rho(p)=\rho_{0}(p, \Theta)+\rho_{6}(p, \Theta) \cos (6 \varphi)+$ $\rho_{12}(p, \Theta) \cos (12 \varphi)$, simulates electron-positron densities in $\mathrm{Gd}$, where the anisotropy is much higher than in $\mathrm{Mg}$ and densities are typical of transition metals. Here we calculated 15 projections $J\left(p_{z}\right)$ with $p_{z}$ along three main and twelve intermediate projections (Fig. 2), changing $\beta$ and $\alpha$ by $15^{\circ}$. In this case our model densities were reproduced in details - see Fig. 3. Here the anisotropic part of reconstructed densities is described by $\sum \rho_{l}(p) P_{l}^{m}(\theta) c_{l}^{m} \cos (m \varphi)$ with $(l, m)=(6,6)$, $(8,6),(10,6),(12,6),(14,6),(12,12),(14,12)$. For this model we show results on the $\Gamma M K$ and $\Gamma^{1} M^{1} K^{1}$ planes where $\Gamma \Gamma^{1}=2 \Gamma A$. On the contrary to the first model, where $\rho(p)$ has similar values in $\Gamma$ and $\Gamma^{1}$, here the densities in the point $\Gamma^{1}$ are about 2.2 times lower than in $\Gamma$ (although there are 3 valence electrons/atom in $\mathrm{Gd}$ while in $\mathrm{Mg}$ only 2). It is connected with the fact that for $\mathrm{Mg}$ valence electrons are almost free and in Gd (transition metal) they have $s+d$ character and there is the contribution of two hole Fermi surfaces in the $\Gamma^{1}$ point in the extended zone scheme. It should be noticed here that contrary to the Compton profiles, our spectra (Fig. 2) represent plane integrals of $\rho(p)$ (with the statistic $7 \times 10^{6}$ counts at peak) with the most contribution of valence states.

Our tests clearly show that this new reconstruction technique [5] works very well. Of course, in order to check if this method is the most efficient indeed, we intend to apply other techniques [1-3] to the same model projections in the future work. 


\section{Acknowledgments}

One of us (M. Samsel) would like to thank the Foundation for Science and Technology (Poland) for financing her participation in the 30th SPA Conference.

\section{References}

[1] P.E. Mijnarends, Phys. Rev. 160, 512 (1967).

[2] N.K. Hansen, HMI-Report B 342, (1980); N.K. Hansen, P. Pattison, J.R. Schneider, Z. Phys. B 66, 305 (1987).

[3] G. Reiter, R. Silver, Phys. Rev. Lett. 54, 1047 (1985).

[4] L. Dobrzyński, A. Holas, Nucl. Instrum. Methods Phys. Res. A 383, 589 (1996).

[5] G. Kontrym-Sznajd, Nukleonika 42, 137 (1997); G. Kontrym-Sznajd, M. Samsel, R.N. West, in preparation.

[6] P.A. Walters, J. Mayers, R.N. West, Positron Annihilation, Eds. P.G. Coleman, S. Sharma, L.M. Diana, North-Holland, Amsterdam 1982, p. 334.

[7] R.L. Waspe, R.N. West, in Ref. [6], p. 242; A. Alam, R.L. Waspe, R.N. West, Positron Annihilation, Eds. L. Dorikens-Vanpraet, M. Dorikens, D. Seger, World Sci., Singapore 1988, p. 242.

[8] A.M. Cormack, J. Appl. Phys. 34, 2722 (1963); 35, 2908 (1964); G. Kontrym-Sznajd, Phys. Status Solidi B 117, 227 (1990)). 